\title{
PRIMORDIAL TORSION FIELDS AS AN EXPLANATION OF THE ANISOTROPY IN COSMOLOGICAL ELECTROMAGNETIC PROPAGATION
}

\author{
ANTONIO DOBADO \\ Departamento de Física Teórica, Universidad Complutense de Madrid, \\ 28040 Madrid, Spain \\ and \\ ANTONIO L. MAROTO \\ Departamento de Física Teórica, Universidad Autónoma de Madrid, \\ 28049 Madrid, Spain
}

Received 24 November 1997

Revised 27 November 1997

\begin{abstract}
In this letter we provide a simple explanation of the recent finding of anisotropy in electromagnetic (EM) propagation claimed by Nodland and Ralston. We consider, as a possible origin of such effect, the effective coupling between EM fields and some tiny background torsion field. The coupling is obtained after integrating out charged fermions, it is gauge-invariant and does not require the introduction of any new physics.
\end{abstract}

In a recent paper, Nodland and Ralston ${ }^{1}$ have reported on a systematic rotation of the plane of polarization of electromagnetic radiation coming from distant radio galaxies, even after Faraday rotation is extracted. Provided that there is no hidden systematic bias in the data or in their analysis, this finding could indicate the presence of some anisotropic background $s^{\mu}$ over large cosmological scales. The authors of Ref. 1 claimed that this effect can be described by a term in the electromagnetic Lagrangian given by

$$
\left(\frac{\Lambda_{s}^{-1}}{4}\right) \varepsilon^{\mu \nu \alpha \beta} F_{\mu \nu} A_{\alpha} s_{\beta}
$$

where $A_{\mu}$ is the electromagnetic potential, $F_{\mu \nu}=\partial_{\mu} A_{\nu}-\partial_{\nu} A_{\mu}, \Lambda_{s}^{-1}$ is a constant of the order of $10^{-32} \mathrm{eV}$ in natural units and $s_{\mu}=(0, \mathbf{s})$ is some phenomenological vector-like parameter with constant unit $\mathbf{s}$. From their analysis of 71 distant galaxies $(z>0.3)$, they found $\mathbf{s}=(\operatorname{decl}, R . A)=.\left(0^{\circ} \pm 20^{\circ}, 21 \pm 2 \mathrm{hr}\right)$.

From the point of view of the physical origin of $s_{\mu}$, the most natural assumption seems to be to consider it as a dynamical vector field. However, as was discussed in Ref. 1, the main problem of that explanation is that the Lagrangian above is not 
gauge-invariant, unless $s_{\mu}$ is kept constant, which is quite unnatural for a dynamical field, or the gradient of some scalar field $\phi, s_{\mu}=\partial_{\mu} \phi$. In this last case, new physics containing this field must be introduced.

After the publication of Ref. 1 there have been several criticisms to the analysis of the data ${ }^{2}$ and responses to them. ${ }^{3}$ Different mechanisms giving rise to anisotropy (such as spontaneous symmetry breaking or universe rotation) have been considered in the literature. ${ }^{4}$ In particular, it is shown that the Nodland and Ralston effect is compatible with a previous observation of the spin axes distribution of certain galaxies.

In any case, the purpose of this letter is to point out that, provided that the effect exists, there is a simple interpretation of the Lagrangian above within the framework of the standard quantum electrodynamics (QED) and classical gravity. In this interpretation, $s_{\mu}$ is understood as the pseudotrace of the torsion field and gauge invariance is preserved without the introduction of any new scalar field $\phi$. In order to prove that, we consider the following simple model to describe the present state of the observable universe. We assume a flat space-time with a classical background torsion field $T^{\mu \nu \alpha}$ (although our results can be generalized to the case when curvature is present in a straightforward way). The mathematical origin of this tensor is the following: consider a pseudo-Riemannian space-time manifold with metric tensor $g_{\mu \nu}$. As usual, in order to define the parallel transport of vectors, we should introduce a new object, an affine connection, whose components are $\hat{\Gamma}_{\mu \nu}^{\lambda}$. Such an arbitrary connection is in principle independent of the metric. However, if we want the lengths and angles of vectors to be invariant under parallel transport, it is needed that the connection be metric, i.e.

$$
\left(\hat{\nabla}_{\lambda} g\right)_{\mu \nu}=\partial_{\lambda} g_{\mu \nu}-\hat{\Gamma}_{\lambda \mu}^{\kappa} g_{\kappa \nu}-\hat{\Gamma}_{\lambda \nu}^{\kappa} g_{\kappa \mu}=0
$$

where $\hat{\nabla}$ is the corresponding covariant derivative. This condition allows us to find the following general form for this kind of connections:

$$
\hat{\Gamma}_{\mu \nu}^{\lambda}=\Gamma_{\mu \nu}^{\lambda}+\frac{1}{2}\left(T_{\nu}^{\lambda}{ }_{\mu}+T_{\mu \nu}^{\lambda}+T_{\mu \nu}^{\lambda}\right)
$$

where the antisymmetric part, $T^{\lambda}{ }_{\mu \nu}=\hat{\Gamma}_{\mu \nu}^{\lambda}-\hat{\Gamma}_{\nu \mu}^{\lambda}$, is known as the torsion tensor and $\Gamma^{\lambda}{ }_{\mu \nu}$ are the usual Christoffel symbols that can be obtained from the metric tensor. In the absence of torsion, any metric connection reduces to the Levi-Civita one, given by the Christoffel symbols. This was the connection considered by Einstein in his formulation of general relativity. However, nowadays the modern theories of gravity consider the metric tensor and the connection as independent entities and therefore torsion appears in a natural way.

By means of the Einstein Equivalence Principle, it is possible to find the minimal Lagrangian for a fermion interacting with a background gravitational field with torsion, ${ }^{5}$ then it is easy to show that only the axial pseudo-trace vector-field $S_{\beta}=\varepsilon_{\mu \nu \alpha \beta} T^{\mu \nu \alpha}$ appears in the minimal coupling. When there is also a background 
electromagnetic field and no curvature, the Lagrangian describing the electrodynamics of different charged fermions $\Psi_{i}$ is given by

$$
\mathcal{L}=-\frac{1}{4} F_{\mu \nu} F^{\mu \nu}+\sum_{i} \bar{\Psi}_{i}\left(i \not D_{i}-m_{i}-\frac{1}{8} \$ \gamma_{5}\right) \Psi_{i}
$$

where $D_{i}^{\mu}=\partial^{\mu}-i e Q_{i} A^{\mu}$ with $m_{i}$ and $Q_{i}$ being the mass and the electric charge of the corresponding fermionic field. Now it is immediate to integrate out the fermionic fields to find the effective action for the electromagnetic field propagating in the torsion classical background:

$$
e^{i S_{\mathrm{eff}}[A, S]}=\int[d \Psi][d \bar{\Psi}] e^{i \int d^{4} x \mathcal{L}},
$$

where $\Psi$ denotes all the fermion fields. By using standard manipulations, it is possible to write:

$$
S_{\mathrm{eff}}[A, S]=-\int d^{4} x\left(\frac{1}{4} F_{\mu \nu} F^{\mu \nu}\right)+i \sum_{i} \operatorname{Tr} \log \left(i \not D_{i}-m_{i}-\frac{1}{8} \not \gamma_{5}\right)
$$

which can be developed as:

$$
\begin{aligned}
S_{\mathrm{eff}}[A, S]= & -\int d^{4} x\left(\frac{1}{4} F_{\mu \nu} F^{\mu \nu}\right) \\
& +i \sum_{i} \sum_{k=1}^{\infty} \frac{(-1)^{k}}{k} \operatorname{Tr}\left[\left(i \not \partial-m_{i}\right)^{-1}\left(e Q_{i} \not A-\frac{1}{8} \not \gamma_{5}\right)\right]^{k} .
\end{aligned}
$$

Computing the functional traces by using dimensional regularization with $D=4-\varepsilon$, it is possible to obtain:

$$
\begin{aligned}
S_{\mathrm{eff}}[A, S]= & -\int d^{4} x\left(\frac{1}{4} F_{\mu \nu} F^{\mu \nu}\right) \\
& -\sum_{i} \frac{e^{2} Q_{i}^{2} m_{i}^{2}}{2} \int d \tilde{q} d \tilde{p} d x d y d z e^{i p(y-x)} e^{i q(z-x)} \Gamma(3-D / 2) \\
& \times \int_{0}^{1} d x_{1} \int_{0}^{x_{1}} d x_{2} \frac{i}{16 \pi^{2}} \varepsilon^{\mu \nu \rho \sigma}\left(A_{\nu}^{y} A_{\rho}^{z} S_{\sigma}^{x}\left(p_{\mu} x_{1}+q_{\mu} x_{2}\right)\right. \\
& \left.+A_{\mu}^{y} A_{\rho}^{z} S_{\sigma}^{x}\left(p_{\nu}\left(x_{1}-1\right)+q_{\nu} x_{2}\right)+A_{\mu}^{y} A_{\nu}^{z} S_{\sigma}^{x}\left(p_{\rho}\left(x_{1}-1\right)+q_{\rho}\left(x_{2}-1\right)\right)\right) \\
& \times F\left(p, q, x_{1}, x_{2} ; m_{i}\right)+\mathcal{O}\left(S^{2}\right)+\mathcal{O}\left(A^{3}\right)
\end{aligned}
$$

with

$$
F\left(p, q, x_{1}, x_{2} ; m_{i}\right)=\left(-m_{i}^{2}+p^{2}\left(x_{1}-x_{1}^{2}\right)+2 p q\left(x_{2}-2 x_{1} x_{2}\right)+q^{2}\left(x_{2}-x_{2}^{2}\right)\right)^{-1},
$$


where $d \tilde{p}=d^{D} p \mu^{\varepsilon} /(2 \pi)^{D}$ and $\mathcal{O}\left(S^{2}\right), \mathcal{O}\left(A^{3}\right)$ denote terms with two or more torsion fields and three or more photon fields respectively. As can be easily seen, this contribution to the effective action is finite. Therefore it is possible to expand the function $F$ in powers of $\mathcal{K}^{2} / m_{i}^{2}$, where $\mathcal{K}$ denotes generically the external momenta. To lowest order we find:

$$
F\left(p, q, x_{1}, x_{2} ; m_{i}\right)=-\frac{1}{m_{i}^{2}}\left(1+\mathcal{O}\left(\frac{\mathcal{K}^{2}}{m_{i}^{2}}\right)\right) .
$$

In this way, we can perform explicitly the integrals in the Feynman parameters $x_{1}$ and $x_{2}$ in (8) order by order, so that we obtain a local expansion for the effective Lagrangian, whose lowest order is given by:

$$
\mathcal{L}_{\text {eff }}=-\frac{1}{4} F_{\mu \nu} F^{\mu \nu}-\frac{\alpha}{24 \pi}\left(\sum_{i} Q_{i}^{2}\right) \varepsilon^{\mu \nu \alpha \beta} F_{\mu \nu} A_{\alpha} S_{\beta}+h d t,
$$

where $\alpha$ is fine structure constant and $h d t$ stands for the higher derivative terms coming from the higher orders in the expansion of the function $F$. At this point it is important to stress that, as far as this model is not anomalous, ${ }^{6}$ the effective action defined in (5) is gauge-invariant. The higher derivative terms in (11) can be neglected at long distances, but are needed to preserve the gauge invariance of the whole effective Lagrangian. Note that, as discussed above, the second term on the R.H.S. of (11) alone is not gauge-invariant unless $S_{\mu}$ were the gradient of some new scalar function. ${ }^{7}$

Thus from the Feynman diagram in Fig. 1 we have found the appropriate term in the Lagrangian in an invariant manner without the introduction of any new scalar field $\phi$, as can be observed by the simple identification

$$
\Lambda_{s}^{-1} s_{\mu}=-\frac{\alpha}{6 \pi}\left(\sum_{i} Q_{i}^{2}\right) S_{\mu} .
$$

For example, by considering the matter content of the standard model we have $\sum_{i} Q_{i}^{2}=N_{f}\left(1+N_{c}(4 / 9+1 / 9)\right)$ which for three families $\left(N_{f}=3\right)$ and three colors $\left(N_{c}=3\right)$ equals 8 . Thus by assuming a space-like $S_{\mu}$ field $\left(S_{\mu}=(0, \mathbf{S})\right)$, the required module for $\mathbf{S}$ to explain the polarization effect observed by Nodland and Ralston should be something about $10^{-30} \mathrm{eV}$.

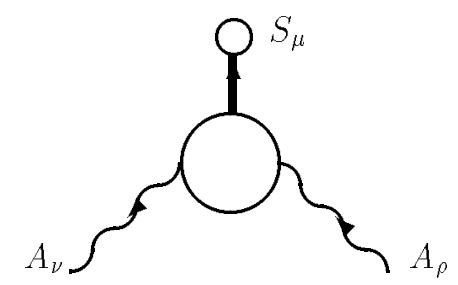

Fig. 1. Feynman diagram contributing to Eq. (11). The double line with a blob represents the external torsion field, the wavy lines the photon fields and in the loop the charged fermions are running represented by the full line. 
Concerning the origin of the torsion field, as commented before, in most of theories of gravitation the affine connection and the vielbein (which is related to the metric tensor), are considered as independent entities and thus torsion appears in a natural way. If the description provided here for the Nodland and Ralston effect were appropriate, it could be the first indication of the presence of torsion at cosmological scales. But even if this were not the case and the effect were not confirmed, this mechanism would allow us to set a limit for the value of a possible cosmological torsion field. From the point of view of a quantum field theory for torsion, the existence of such field probably requires that $S_{\mu}$ be massless or having a vacuum expectation value. This could be in contradiction with the usual assumptions of low-energy effective quantum gravity in which the torsion field is supposed to have a mass of the order of Planck mass. ${ }^{8}$ Despite that and provided that this new effect is confirmed, we really believe that this is the most economical explanation of the finding by Nodland-Ralston since it does not require the introduction of new fields, apart from those appearing in the standard model and our current description of classical gravity.

\section{Acknowledgments}

We are grateful to B. Nodland for his comments to the preliminary version of this letter and also to J. P. Ralston for his suggestions. This work has been supported in part by the Ministerio de Educación y Ciencia (Spain) (CICYT AEN96-1634).

\section{References}

1. B. Nodland and J. P. Ralston, Phys. Rev. Lett. 78, 3043 (1997).

2. D. J. Eisenstein and E. F. Bunn, astro-ph/9704247; S. M. Carroll and G. B. Field, Phys. Rev. Lett. 79, 2394 (1997); J. P. Leahy, astro-ph/9704285; J. F. C. Wardle, R. A. Perley and M. H. Cohen, Phys. Rev. Lett. 79, 1801 (1997); T. J. Loredo, E. E. Flanagan and I. M. Wasserman, astro-ph/9706258.

3. B. Nodland and J. P. Ralston, Phys. Rev. Lett. 79, 1958 (1997); astro-ph/9706126; astro-ph/9708114.

4. D. V. Ahluwalia and T. Goldman, Mod. Phys. Lett. A8, 2623 (1993); R. W. Kuhne, ibid. A12, 2473 (1997).

5. F. W. Hehl, P. von der Heyde, G. D. Kerlick and J. M. Nester, Rev. Mod. Phys. 48, 393 (1976).

6. A. Dobado and A. L. Maroto, Phys. Rev. D54, 5185 (1996).

7. V. De Sabbata and M. Gasperini, Phys. Lett. A83, 115 (1981).

8. S. M. Carroll and G. B. Field, Phys. Rev. D50, 3867 (1994); R. T. Hammond, ibid. D52, 6918 (1995). 